\title{
Pain-related somatosensory evoked potentials in cortical reflex myoclonus
}

Ryusuke Kakigi, Hiroshi Shibasaki, Ryuji Neshige, Akio Ikeda, Kousuke Mamiya, Yasuo Kuroda

\begin{abstract}
To elucidate the sensitivity to pain stimuli in patients with cortical reflex myoclonus, pain-related somatosensory evoked potentials (pain SEPs) following $\mathrm{CO}_{2}$ laser stimulation and conventional electrically-stimulated SEPs (electric SEPs) were compared in four patients with cortical reflex myoclonus. The P25 peak of electric SEPs was considerably enhanced but the P320 potential of pain SEPs was of normal amplitude in all patients. After medication, myoclonus was reduced and the amplitude of P25 was decreased, but P320 showed no change. In our previous study of the scalp distribution in normal subjects, a subcortical site, probably the thalamus, was considered to be the generator source of P320. Because most pain stimuli do not reach the cortex, patients with cortical reflex myoclonus are not sensitive to pain stimuli and $\mathbf{P 3 2 0}$ in pain SEPs is not enhanced.
\end{abstract}

There is a general concept that cortical reflex myoclonus $^{1}$ is induced mainly by muscle stretch, touch or pressure. ${ }^{2-5}$ This is compatible with the facts that cortical components of electrically-stimulated somatosensory evoked potentials (electric SEPs), which are generated in the sensorimotor cortex as a result of ascending signals mediated through large myelinated fibres, are considerably enhanced ("giant") in most patients with cortical reflex myoclonus. ${ }^{1-10}$ These patients might be sensitive to pain stimulation as well, but by using a pin or needle, it is difficult to give "pure" pain stimuli without causing excitation of cutaneous mechanoreceptors. A low power and long wavelength $\mathrm{CO}_{2}$ laser beam induces pain or heat sensation when applied to the skin. Recently, we have investigated pain-related SEPs (pain SEPs) by $\mathrm{CO}_{2}$ laser stimulation, and as a result of various studies involving ischaemic or anaesthetic conditions in normal subjects, its ascending signals were considered to be mediated through $\mathrm{A} \delta$ fibres. ${ }^{11}$ Bromm and Treede using the microneurogram reached a similar conclusion. ${ }^{12}$

The object of this investigation was therefore to elucidate the degree of sensitivity to pain stimuli in cortical reflex myoclonus by analysing pain SEPs.

\section{Materials and methods}

Four patients with cortical reflex myoclonus, one with Ramsay Hunt syndrome, one with sialidosis type 2 , and 2 with unclassified progressive myoclonic epilepsy, were selected according to the following three criteria; ${ }^{9}(1)$ the amplitude of the P25, the initial cortical positive potential recorded at or around the somatosensory hand area of the scalp following stimulation of the median nerve at the wrist, was larger than the mean $+2 \mathrm{SD}$ for normal subjects. (2) An enhanced long-loop reflex (C reflex ${ }^{13}$ ) recorded even at rest (without voluntary contraction of the corresponding muscles), and (3) the presence of cortical spikes preceding spontaneous myoclonic jerks using the jerk-locked back averaging method. ${ }^{7814}$ The patients' profiles are summarised in table 1 . No neurological dysfunction other than myoclonus, cerebellar ataxia or generalised convulsion was found. Thirty normal subjects (10 females and 20 males, mean age 46 years, range 18 to 75 ) were also studied. All subjects gave informed consent.

In addition to using the conventional pinprick method, pain sense was also evaluated by a specially made dolorimeter using a $\mathrm{CO}_{2}$ laser beam, to determine the exact strength of stimulus. Laser wavelength was $10.6 \mu \mathrm{m}$, and the diameter of the irradiated beam was approximately $2 \mathrm{~mm}$. The stimulus was applied to the dorsum of the hand, with increasing power, once every 3 seconds. Subjects were requested to tell the examiner each time they felt mild touch (pre-pain threshold) and distinct sharp pain "like a pin-prick" (pain threshold). As the intensity above $20.5 \mathrm{~mJ} / \mathrm{mm}^{2}$ was suspected to cause skin erythema, maximum power was set to that level. The test was repeated at least three times and the lowest threshold was adopted.

The electric stimulus was a constant voltage square-wave pulse of $0.2 \mathrm{~ms}$ duration delivered to the left median nerve at the wrist. The stimulus intensity was sufficient to produce a definite twitch of the thumb.

Stimulus intensity of approximately 17$20 \mathrm{~mJ} / \mathrm{mm}^{2}$ elicited sharp pain that subjects tolerated without any discomfort and felt "like a pin-prick". Stimulus duration was $10 \mathrm{~ms}$. To avoid habituation, irradiated sites were changed slightly from stimulus to stimulus. A red helium-neon laser was combined with the $\mathrm{CO}_{2}$ laser to indicate the irradiated area. The temporal delay between laser stimulation and the onset of averaging was less than $1 \mathrm{~ms}$. The detailed methods were described in our previous study. ${ }^{11}$ 
Table 1 Clinical profiles of four cases of cortical reflex myoclonus

\begin{tabular}{lllll}
\hline Case & 1 & 2 & 3 & 4 \\
\hline Sex & F & F & M & M \\
Age (years) & 19 & 71 & 54 & 52 \\
Age at onset & 10 & 24 & 30 & 45 \\
Diagnosis & Ramsay Hunt syndrome & PME` & PME & Sialidosis type 2 \\
Generalised convulsion & $(+)$ & $(+)$ & $(+)$ & $(-)$ \\
Cerebellar signs & $(+)$ & $(-)$ & $(-)$ & $(+)$ \\
\hline
\end{tabular}

«Unclassified progressive myoclonic epilepsy

Recording was performed in a warm and quiet room. The patient was seated comfortably in a reclining arm-chair, and was encouraged to relax. Skin temperature of the stimulated area was measured by a thermometer. The skin was warmed by a heater when it was below $30^{\circ} \mathrm{C}$. The patient was awake during the recording.

Silver disc recording electrodes were attached at 16 locations on the scalp, each of which corresponded to the midway between the conventional electrode locations of the international $10-20$ system (fig 1 ). The reference used was the left earlobe for electric SEPs and linked earlobes for pain SEPs.

The analysis window of pain SEPs was $200 \mathrm{~ms}$ for recording short-latency components and $1500 \mathrm{~ms}$ for recording longer ones, and that of electric SEPs was from $10 \mathrm{~ms}$ before the stimuli to $90 \mathrm{~ms}$ after the stimuli. The frequency response of the amplifier was 1$1000 \mathrm{~Hz}(-3 \mathrm{~dB})$ for recording electric SEPs and short-latency pain SEPs, and $0.5-100 \mathrm{~Hz}$ for long-latency pain SEPs. The number of sampling points was 512 for each channel. Two hundred to 300 responses were averaged for electric SEPs and short-latency pain SEPs, and 30 to 100 for long-latency pain SEPs. At least two recordings were made for each stimulus condition. Relative positivity at grid 1 resulted in a downward deflection in all recordings. Amplitude was measured from the preceding peak of the opposite polarity wave.

The isopotential maps were drawn using the amplitude measured from baseline at each electrode at a particular time interval after the stimulus onset. The baseline was determined by averaging the $10 \mathrm{~ms}$ epoch before the stimuli for electric SEPs, and the $100 \mathrm{~ms}$ epoch after the stimuli for pain SEPs.

Figure 1 Electrode location was midway between the conventional electrode locations of the 10-20 system. For example, location 6 was midway between $\mathrm{Fz}$ and $\mathrm{Cz}$.
Long-loop reflex (C reflex) was recorded from a pair of surface electrodes placed on the thenar muscle of the stimulated side, while it was kept at rest throughout the recording. The frequency response of the amplifier was between 75 and $1000 \mathrm{~Hz}$, and the analysis window was $100 \mathrm{~ms}$ following electrical stimulation of the median nerve and 1500 or $2500 \mathrm{~ms}$ following laser stimulation.

The recordings were carried out when the patients were not taking medication, and were performed again when they were on appropriate medication, to discover any drug effects.

\section{Results}

\section{(1) Pain threshold}

Pre-pain and pain thresholds in patients were not significantly different from those in normal subjects (table 2). No patient felt hyperalgesia or dysalgesia to laser stimuli, and myoclonus was not induced by laser stimuli.

\section{(2) Pain SEPs}

In normal subjects the N200-P320-N500 complex was clearly identified in pain SEPs, ${ }^{11}$ but no potential earlier than $\mathrm{N} 200$ could be found. As the P320 potential was the largest and most stable one with relatively small inter-individual differences, P320 was evaluated in this study. Although mean peak latency of P320 was $331 \cdot 6$ $\mathrm{ms}$ in the present study, it was termed P320 instead of P330, following the nomenclature used in our previous study. ${ }^{11}$

As in the normal subjects, short-latency potential was not recorded in the patients. The P320 was clearly identified in all patients, and was of normal latency and amplitude (table 3 and fig 2A). It was maximal at the location 6 (midway between $\mathrm{Fz}$ and $\mathrm{Cz}$ ) in cases 3 and 4, and at the location 11 (midway between $\mathrm{Cz}$ and $\mathrm{Pz}$ ) in cases 1 and 2 . P320 was widely distributed to the surrounding area, especially in the frontal region, with no significant asymmetry (fig 3A). No negative potential which appeared to show phase-reversal to $P 320$ could be identified. Scalp topography of P320 was similar to that recorded in normal subjects. ${ }^{11}$

Long-latency EMG response from the thenar muscle of the stimulated hand was not identified in any of the cases.

(3) Electric SEPs

P25 potential in electric SEPs was considerably enhanced in all cases compared with those in normal subjects (table 3 and fig 2B). The P25 was maximal around the somatosensory hand area, that is, at the location 12 (midway between $\mathrm{C} 4$ and P4) in cases 2-4 (fig 3B), and at the location 7 (midway between F4 and C4) in

Table 2 Pre-pain and pain thresholds judged by the dolorimeter using $\mathrm{CO}_{2}$ laser.

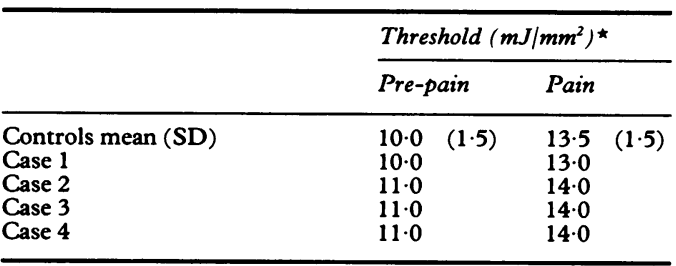

ॠThreshold of the dorsum of the left hand. 
Table 3 P320 peak of pain SEP and P25 of electric SEP in patients before and after medication and in normal controls

\begin{tabular}{|c|c|c|c|c|c|c|c|c|c|}
\hline \multirow{2}{*}{$\frac{\text { Case }}{\text { Medication }}$} & \multirow{2}{*}{\multicolumn{2}{|c|}{$\begin{array}{l}1 \\
\text { Clonazepam, } 6 \mathrm{mg} \\
\text { Valproate, } 800 \mathrm{mg} \\
\text { Primidone, } 500 \mathrm{mg}\end{array}$}} & \multirow{2}{*}{\multicolumn{2}{|c|}{$\begin{array}{l}2 \\
\text { Clonazepam, } 6 \mathrm{mg} \\
\text { Valproate, } 400 \mathrm{mg}\end{array}$}} & \multirow{2}{*}{\multicolumn{2}{|c|}{$\frac{3}{\text { Clonazepam, } 6 \mathrm{mg}}$}} & \multicolumn{2}{|l|}{4} & \multirow{3}{*}{$\begin{array}{l}\text { Normal controls } \\
\text { mean }(S D)\end{array}$} \\
\hline & & & & & & & Clonaz & am, $3 \mathrm{mg}$ & \\
\hline & Before & After & Before & After & Before & After & Before & After & \\
\hline $\begin{array}{l}\text { P320 Latency (ms) } \\
\text { Amplitude }(\mu \mathrm{V}) \\
\text { P25 Latency } \\
\text { Amplitude }\end{array}$ & $\begin{array}{r}375 \\
3 \cdot 8 \\
26 \cdot 9 \\
14 \cdot 2\end{array}$ & $\begin{array}{r}370 \\
3 \cdot 5 \\
27 \cdot 2 \\
10 \cdot 3\end{array}$ & $\begin{array}{r}285 \\
5 \cdot 0 \\
26 \cdot 0 \\
10 \cdot 2\end{array}$ & $\begin{array}{r}290 \\
5 \cdot 0 \\
25 \cdot 8 \\
8 \cdot 4\end{array}$ & $\begin{array}{r}345 \\
3 \cdot 5 \\
25 \cdot 0 \\
14 \cdot 7\end{array}$ & $\begin{array}{r}350 \\
3.0 \\
25 \cdot 0 \\
11.0\end{array}$ & $\begin{array}{l}340 \\
9 \cdot 0 \\
25 \cdot 2 \\
21 \cdot 6\end{array}$ & $\begin{array}{r}345 \\
7 \cdot 3 \\
25 \cdot 0 \\
12 \cdot 7\end{array}$ & $\begin{array}{rr}331 \cdot 6 & (22 \cdot 1) \\
7 \cdot 1 & (4 \cdot 5) \\
25 \cdot 1 & (2 \cdot 6) \\
4 \cdot 2 & (2 \cdot 2)\end{array}$ \\
\hline
\end{tabular}

case 1 . The negative component, N25, which could be identified in the frontal region, was also very large in all cases. Scalp topography of P25 was very similar to that in normal subjects. The following negative potential, N34, was also very large, particularly in cases 2 and 3 (fig 2B).

Clear $\mathrm{C}$ reflex was identified in all cases and its onset latency was between 44 and $49 \mathrm{~ms}$.

(4) Drug effects on SEPs

While patients were on an appropriate medication such as clonazepam, primidone and sodium valproate, myoclonus was remarkably reduced in all cases. Amplitude of P25 and N34 in electric SEPs was also significantly decreased, and $C$ reflex could not be recorded any more in all cases. However, the P320 in pain SEPs showed no remarkable change in all cases (table 3 and fig 4).

\section{Discussion}

Several papers have described pain SEPs following $\mathrm{CO}_{2}$ laser stimulation in normal subjects, ${ }^{111215-20}$ but this is the first report of pain SEPs in cortical reflex myoclonus, which is characterised by the so-called giant SEPs following electrical stimulation.

Although generator mechanisms of the giant electric SEPs are not yet fully understood, hyperexcitability of or decreased inhibitory mechanisms of neurons in the sensorimotor cortex is believed to be a contributing factor. Therefore, the normal pain SEPs in cortical reflex myoclonus in this study suggest either that impulses arising from pain-temperature stimuli do not reach the cortex, or that neuronal excitability in response to pain stimuli is not enhanced in the sensory cortex. However, in view of the scalp distribution of P320 which was maximal around the vertex in our study, it is difficult to adopt the latter hypothesis.

In primates the primary sensory cortex (SI) can now be subdivided into four regions, corresponding to cortical areas $1,2,3 a$ and $3 b .^{2}$ Area $3 a$ has been shown to be a receiving area for muscle spindle afferents, ${ }^{22}$ and is therefore assumed to be concerned with the processing of proprioceptive information. Area 2 has been found to be responsive to stimulation of "deep" (non-cutaneous) body tissues, ${ }^{23} 24$ and therefore is also likely to be mainly concerned with proprioception. Units in areas 1 and $3 b$, however, respond mainly to cutaneous stimuli. ${ }^{21} 2325$ In contrast, cortical columns or neurons that process temperature or noci-
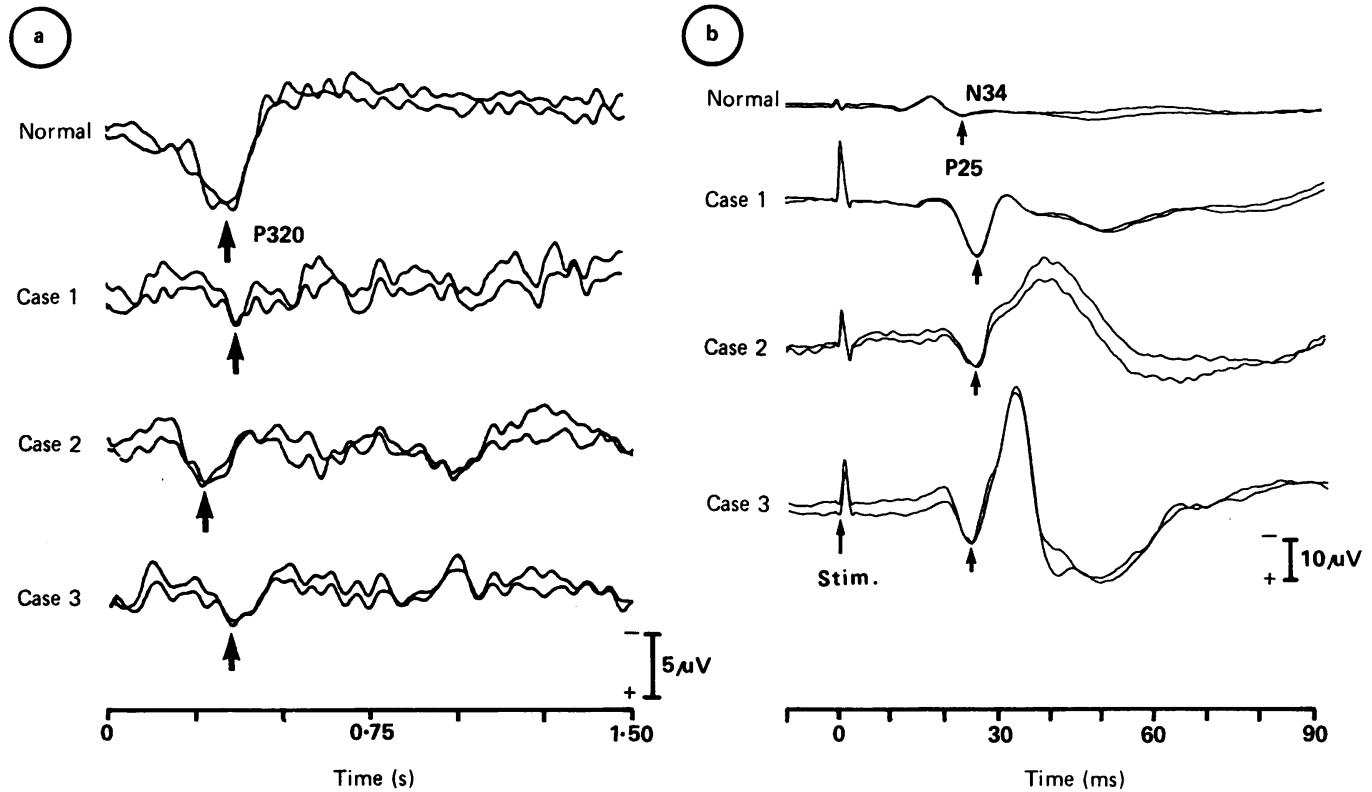

Figure 2 Waveforms of pain SEPs following $\mathrm{CO}_{2}$ laser stimulation of the left hand $(A)$ and electric SEPs following stimulation of the left median nerve at the wrist $(B)$ in cases 1-3 of cortical reflex myoclonus and in a normal subject to show the nomenclature of each recognisable peak. The P320 potential of pain SEPs indicated by arrows is of normal amplitude $(A)$, but P25 in electric SEPs indicated by small arrows is remarkably enhanced ( $B$ ) in all cases. The active electrode is placed at location 6 (midway between $F z$ and $C z$ ) for pain $S E P s$, and at location 12 (midway between C4 and P4) for electric SEPs. The reference was the linked earlobes in pain SEPs and the left earlobe in electric SEPs. Results in case 4 are shown in fig 4. 
Figure 3 Scalp topography of the $P 320$ of the pain $S E P(A)$ and $P 25$ of the electric $S E P$ $(B)$ in case 3. P320 was maximal at the midfrontal area and widely distributed, and P25 was maximal at the right somatosensory hand area.

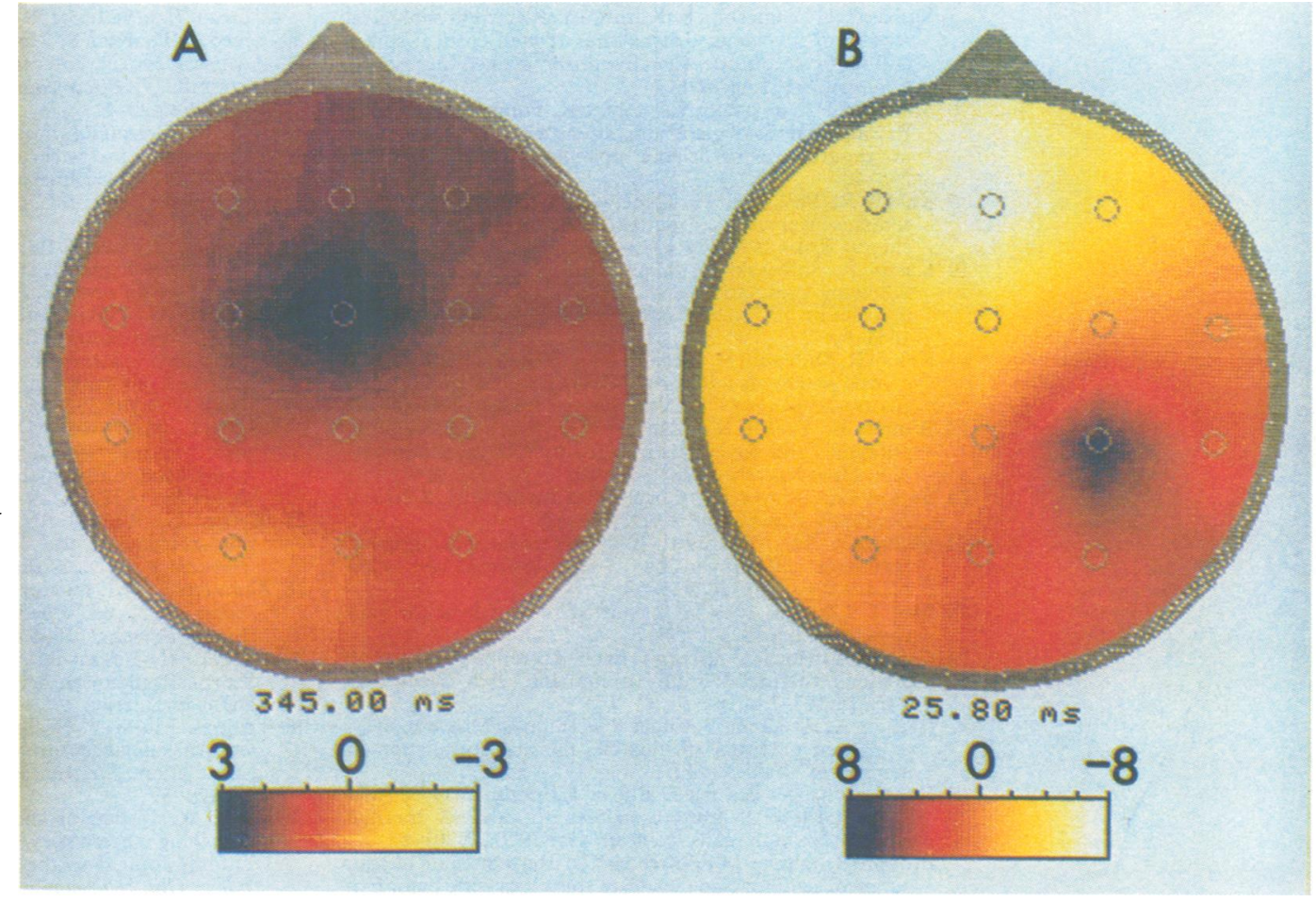

ceptive stimuli have not yet been definitely identified. ${ }^{26}$

In our previous study of pain SEPs in normal subjects, ${ }^{11}$ we proposed the hypothesis that the P320 might be generated in the subcortical area, probably thalamus, as a result of ascending signals mediated through $\mathrm{A} \delta$ fibres. The anatomical findings described above and the present results in which pain SEPs were not enhanced even in cortical reflex myoclonus support our hypothesis and agrees with the general concept that cortical reflex myoclonus is usually not induced by pure pain or thermal stimulation.

The generator source of the P25 in electric SEPs is generally considered, because of its scalp distribution, to be area 1 or $2,^{2728}$ or $3 b^{29}$ as a result of ascending signals mediated through large myelinated fibres. This hypothesis supports the fact that P25 is considerably enhanced in cortical reflex myoclonus as well as with the fact that cortical reflex myoclonus is generally induced by muscle stretch, touch or pressure.

Following medication, myoclonus was remarkably reduced and amplitude of P25 was markedly decreased, but P320 showed no significant change. This also supports the view that pain SEPs have little to do with excitability of the cortical neurons.

This study was supported by 'Grant in Aid for Encouraging Young Doctors, No 61770559 and 63770541 ' for RK and 'Grant in Aid for Scientific Research, No 63570368' for HS from The Ministry of Education, Science and Culture, Japan.

The dolorimeter and stimulator for recording SEPs by $\mathrm{CO}_{2}$ laser beam were made by Nippon Infrared Industries Co Ltd, Tokyo, Japan.

1 Hallett M, Chadwick D, Marsden CD. Cortical reflex myoclonus. Neurology 1979;29:1107-25.

2 Dawson GD. Investigations on a patient subject to myoclonic seizures after sensory stimulation. J Neurol Neurosurg Psychiatry 1947;10:141-62.

3 Rosén I, Fehling C, Sedgwick M, Elmqvist D. Focal reflex epilepsy with myoclonus: electrophysiological investigation and therapeutic implications. Electroencephalogr Clin Neurophysiol 1977;42:95-106.

4 Obeso JA, Rothwell JC, Marsden CD. The spectrum of cortical myoclonus: from focal reflex jerks to spontaneous motor epilepsy. Brain 1985;108:193-224. Electrophysiological and positron emission studies in a Electophos tinua and motor epilepsy. J Neurol Neurosurg Psychiatry 1986:49:796-807.

6 Halliday AM. The electrophysiological study of myoclonus in man. Brain 1967;90:241-84.
5 Cowan JMA, Rothwell JC, Wise RJS, Marsden CD.
Figure 4 Drug effects on SEPs in case 4. Following medication, the P320 in pain SEPs showed no remarkable change $(A)$, but the P25 in electric SEPs was much reduced (B). Electrode derivation is location 6-A1A2 in pain $S E P s$ and location 12-A1 in electric $S E P$.
Pain SEPs

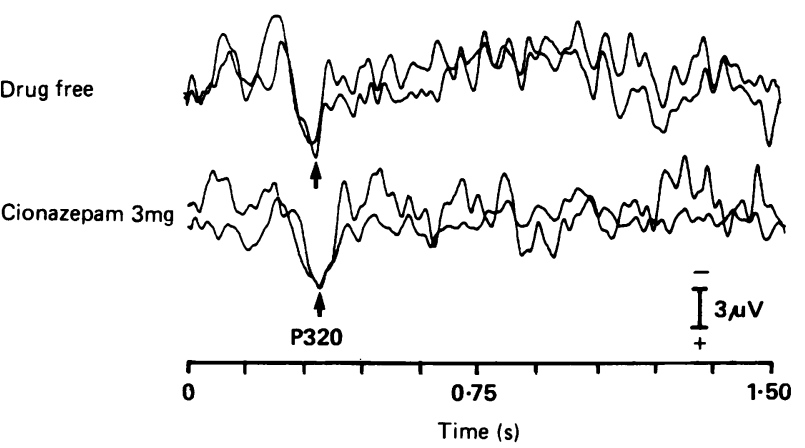

Electric SEPs

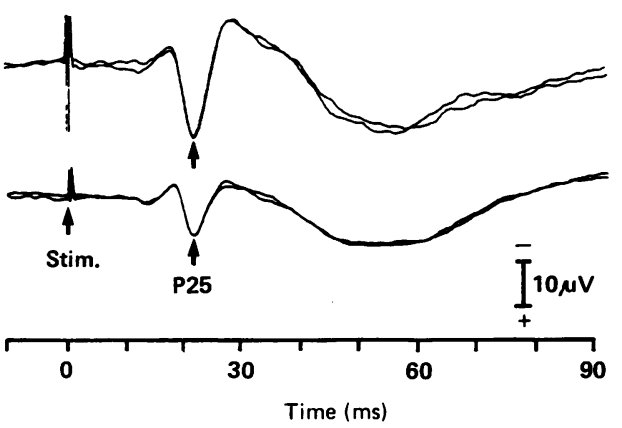


7 Shibasaki H, Yamashita Y, Kuroiwa Y. Electrophysiological studies of myoclonus: myoclonus-related cortical spikes studies of myoclonus: myoclonus-related cortical spikes and high amplitude so

8 Shibasaki H, Yamashita Y, Neshige R, Tobimatsu S, Fukui $R$. Pathogenesis of giant somatosensory evoked potentials in progressive myoclonic epilepsy. Brain 1985;108:

9 Kakigi R, Shibasaki H. Generator mechanisms of gian somatosensory evoked potentials in cortical reflex myoclonus. Brain 1987;110:1359-73.

10 Kakigi R, Shibasaki H. Somatosensory evoked potentials following stimulation of the lower limb in cortical reflex myoclonus. J Neurol Neurosurg Psychiatry 1987;50: 1641-6.

11 Kakigi R, Shibasaki H, Ikeda A. Pain-related somatosensory evoked potentials following $\mathrm{CO}_{2}$ laser stimulation in man. Electroencephalogr Clin Neurophysiol 1989;74:139-46.

12 Bromm B, Treede RD. Nerve fibre discharges, cerebral potentials and sensations induced by $\mathrm{CO}_{2}$ laser stimulapotentials and sensations induced

13 Sutton GG, Mayer RF. Focal reflex myoclonus. J Neurol Neurosurg Psychiatry 1974:37:207-17.

14 Shibasaki H, Kuroiwa Y. Electroencephalographic correlates of myoclonus. Electroencephalogr Clin Neurophysiol 1975;39:455-63.

15 Mor J, Carmon A. A laser emitted radiant heat for pain research. Pain 1975;1:233-7.

16 Carmon A, Mor J, Goldberg J. Evoked cerebral responses to noxious thermal stimuli in humans. Exp Brain Res 1976;25:103-7.

17 Carmon A, Friedman Y, Coger R, Kenton B. Single analysis of evoked potentials to noxious thermal stimulation in man. Pain 1980;8:21-32.

18 Kenton B, Coger R, Crue B, Pinsky J, Friedman Y, Carmon A. A peripheral fibre correlates to noxious thermal A. A peripheral fibre correlates to noxious therm

19 Bromm B, Jahnke MT, Treede RD. Responses of human cutaneous afferents to $\mathrm{CO}_{2}$ laser stimuli causing pain. Exp
Brain Res 1984;55:158-66.

20 Treede RD, Kief S, Holzer T, Bromm B. Late somatosensory evoked potentials in response to cutaneous heat stimuli. Electroencephalogr Clin Neurophysiol 1988;70:429-41.

21 Kaas JH, Nelson RJ, Sur M, Merzenich MM. Organization of somatosensory cortex in primates. In: Schmitt FO, Worden FG, Adelman G, Dennis SG, eds. The Organization of the Cerebral Cortex. Cambridge, MA: MIT Press, 1981:237-61.

22 Phillips CG, Powell TPS, Wiesendanger M. Projection from ow threshold muscle afferents of hand and forearm to area 3a of baboon's cortex. J Physiol (Lond) 1971;217:419-46.

23 Powell TPS, Mountcastle VB. Some aspects of the functional organisation of the cortex of the postcentral gyrus of the monkey: a correlation of findings obtained in a single unit analysis with cytoarchitecture. Bull Johns Hopkins Hosp 1959;105:133-62.

24 Paul RL, Merzenich M, Goodman H. Representation of slowly and rapidly adapting cutaneous mechanoreceptors of the hand in Brodman's area 3 and 1 of Macaca mulatta. Brain Res 1972;36:229-49.

25 Werner G, Whitsel BL. Functional organisation of the somatosensory cortex. In: Iggo A, ed. Handbook of Sensory Physiology, Vol 2, Berlin: Springer, 1973:621-700.

26 Zimmermann $M$. Neurophysiology of sensory systems. In: Schmidt F, ed. Fundamentals of Sensory Physiology, Berlin: Springer, 1986:68-116.

27 Lueders H, Lesser RP, Hahn J, Dinner DS, Klem G. Cortical somatosensory evoked potentials in response to hand stimulation. $J$ Neurosurg 1983;58:885-94.

28 Tsuji S, Murai Y. Scalp topography and distribution of cortical somatosensory evoked potentials to median nerve stimulation. Electroencephalogr Clin Neurophysiol 1986; 65:429-39.

29 Kakigi R. Ipsilateral and contralateral SEP components following median nerve stimulation: effects of interfering stimuli applied to the contralateral hand. Electroencephalogr Clin Neurophysiol 1986;64:246-59. 